\title{
Enhancement of Infra-Red Images of Human Hand
}

\author{
Anup Pillai, Sam Mil'shtein* \\ Advanced Electronic Technology Center, ECE Dept., UMass, Lowell, MA-01854
}

\begin{abstract}
Among the various technologies used for medical imaging, Infra-Red methods occupies very important place, as IR light interaction with human body became to be a base for thermography, tomography and visualization of blood vessels. Different types of medical imaging techniques exists which are classified on the basis of the wavelength of the radiation used, the mode of operation (transmission or reflection) and the reproduction of the acquired information in the form of images. The human hand is one of the most complicated mechanical -biological objects. The complexity of various bones and tendons, joints and muscles, various soft tissues and network of blood vessels presents a significant challenge to any visualization technique. The request of medical professionals for clarity of hand images requires elimination of some parts of the image, colorizing, and contrast enhancement. In this study, we report significant improvement of IR images of the hand. Enhanced IR images allow clear visualization of tendons which are otherwise seen only by MRI.
\end{abstract}

Keywords Infra-Red Imaging, Human Hand, Image Processing

\section{Introduction}

NIR imaging has various applications like optical tomography[1-2], diagnosis of brain edema[3-4] and also mammography[5-6]. IR wavelength range starts from $750 \mathrm{~nm}$ and extends up to $1 \mathrm{~mm}$, covering almost three orders of magnitude in the overall electromagnetic spectrum. However, we are experiment only in Near Infrared (NIR) from 680 $1000 \mathrm{~nm}$. The major content of human tissue is water. Lean muscle tissue contains about $75 \%$ water, blood contains $83 \%$ water, body fat contains $25 \%$ water and bone has $22 \%$ water[7].Interaction of NIR with biological tissues which has low absorption of water can produce interesting potential results.

Considering all of the advantages of near infrared imaging, recent study[8] was initiated to examine human palm by IR light. In this study, bones, tendons, cartilages, and other tissues in the human hand were observed. Every observed element carries a complicated contrast difference. For example, the blood vessels have a different intensity than the tendons; the tendons have different intensity than the soft tissues etc. The near infrared images show many subcutaneous parts like bones, tendons, cartilages, soft tissues etc.

\section{Image Processing Algorithms}

Figure 1 shows a near infrared image of a human palm.

* Corresponding author:

sam_milshtein@uml.edu(Sam Mil'shtein)

Published online at http://journal.sapub.org/ajbe

Copyright (C) 2011 Scientific \& Academic Publishing. All Rights Reserved
As seen from the figure, the blood vessels and the tendons appear dark in color in contrast to the surrounding tissues that appear bright. Maximum amount of photons are absorbed by blood which is a mixture of oxygenated hemoglobin and water. Due to the large amount of scattering[9] and complexity of human hand, formation of good contrast becomes difficult. Tendons are present very close to the top surface of the palm. This makes the tendons appear dark in the final output image. Human wrist, being thicker than palm, shown in Figure 2, demonstrates good IR penetration and produces a detailed subcutaneous image. Fourier Transform based algorithm is developed in MATLAB which combines two images taken at two different wavelengths and produces a much enhanced final image as shown in Figure 3 a) - c). In order to take the two images at different wavelengths, a collimator and notch filter assembly is used to provide a much narrow response from the emitter. A 2-D Fourier transformation is applied to both the images and the resulting magnitudes and phases are added. An inverse 2-D Fourier transform of the addition results in the brighter image showing much more details than the original two images. Addition in Fourier domain is better than conventional addition of two images as the process involves less addition of noise[10].

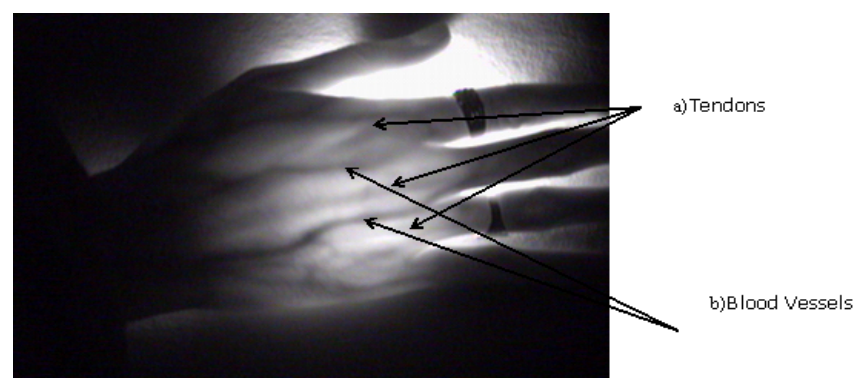

Figure 1.NIR image of Human hand. 


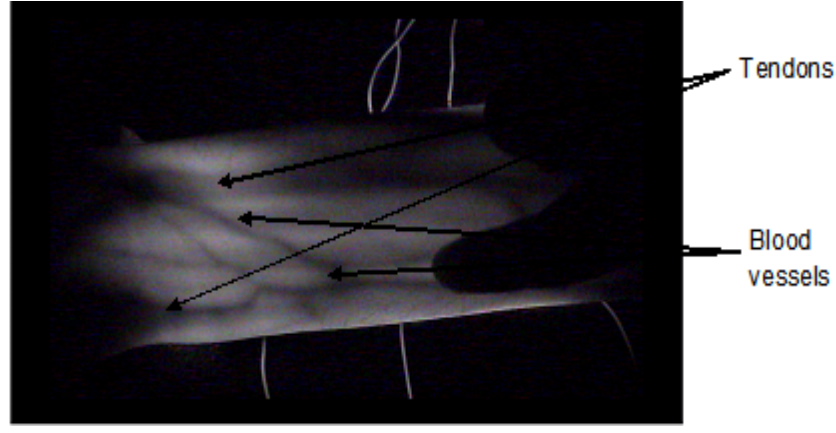

Figure 2. NIR image of Human wrist.

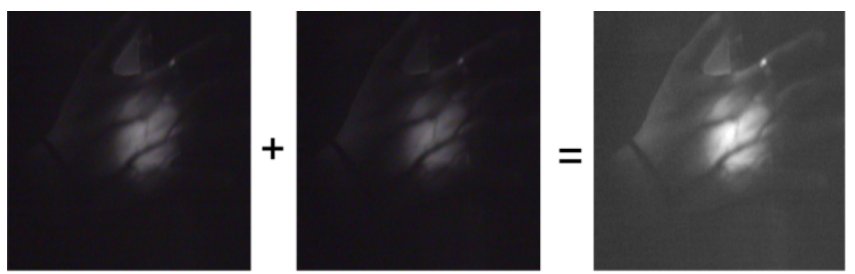

Figure 3. a) Image taken at $780 \mathrm{~nm}$.b)Image taken at $870 \mathrm{~nm}$.c) Fourier addition.

The major application of this technology being detection of tendons, the high dark contrast of the blood vessels is not desirable. There are various image processing techniques developed using MATLAB which allow to enhance the contrast of the tendons and decrease the contrast of the blood vessels.

\subsection{Image Enhancement Algorithm}

Sometimes there is a need to focus on a given part of the visible tendon. The image enhancement algorithm increases the contrast of the region of interest from the surrounding region in order to highlight that specific area.The enhanced image is obtained by recording the image, inverting it, performing a histogram equalization to reduce the range of pixel intensities and then taking an absolute difference of the two images. Figure $4 \mathrm{a}$ and $\mathrm{b}$, illustrates the results of this algorithm.

\subsection{Bleaching Algorithm}

The enhanced image algorithm allows a better visualization of the tendons but the selection of region of interest has to be narrow. In order to overcome this, the bleaching algorithm is used. The enhanced image is again read and stored into a matrix form. The enhanced image is again inverted. Then an absolute difference of the enhanced image and its complement is calculated as shown in Figure 5, a and b. The resultant image has the blood vessels completely bleached out. Due to the alteration of pixel values by performing absolute difference operations, there is a slight amount of blur introduced in the image. This blur is removed by using Weiner filtering[11].

\subsection{Coloring of Blood Vessels Contour Algorithm}

The bleaching of blood vessels algorithm works very well by enhancing the contrast of the tendons. The image would be much better if majority of the blood vessels forming contours in the region of the tendons have a different color in the image. This is achieved by applying the coloring of blood vessels contour algorithm. In this algorithm the image is read as an input and stored in matrix form using MATLAB. Then a raster scan is performed on the elements of the matrix determining the exact value of intensity of each pixel in the image. Now the range of the intensity values of the blood vessels is determined and these values are different from the intensity values of the tendons which are less dark than the blood vessels. The pixels whose matrix elements have the intensity values in the blood vessels range are filtered out. The RGB values of these pixels are adjusted such that these pixels take a certain color and a final colored blood vessels image is obtained. Figure 6 , $a$ and $b$, shows the results of the coloring algorithm.
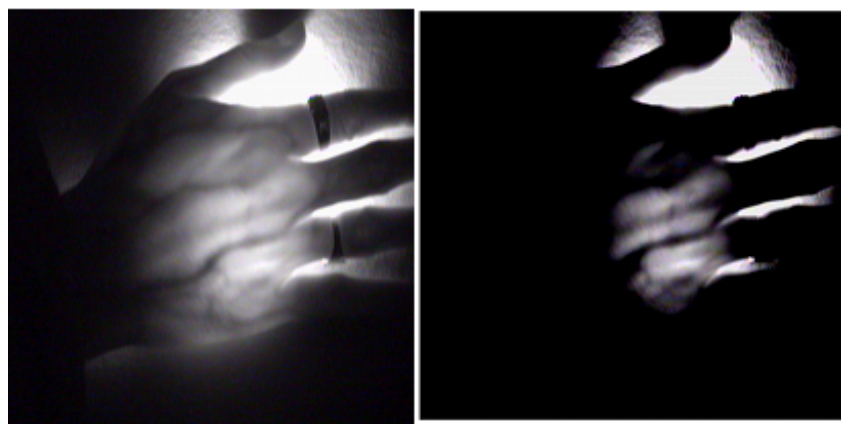

Figure 4. a) - Original Image. Figure 4. b) - Enhanced image.
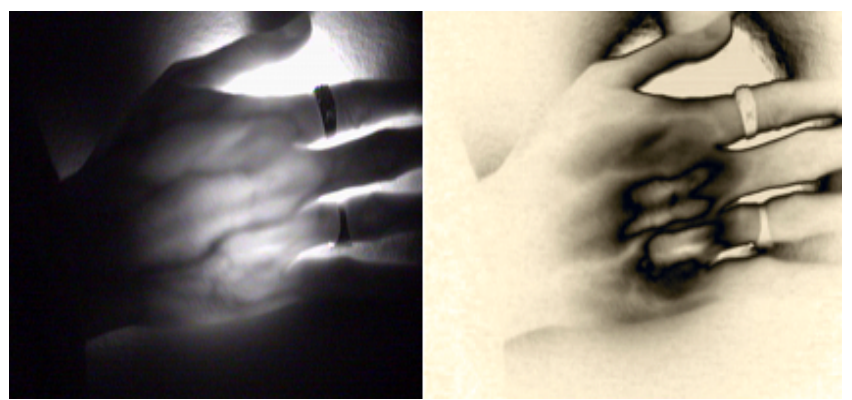

Figure 5. a) Original Image. Figure 5. b) Bleached image.
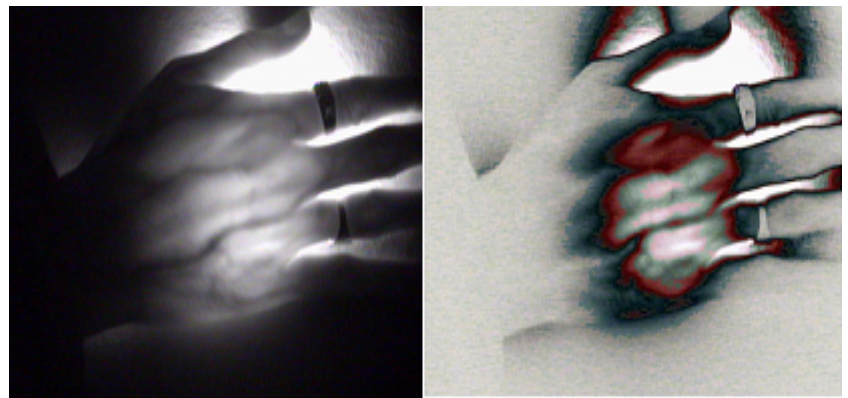

Figure 6. a) Original Image. Figure 6. b) Colorized image.

\section{Conclusions}

Almost all of the NIR imaging techniques and image processing for the human hand is focused towards vein pattern biometrics[12-13]. However, in the manuscript, we have demonstrated that this technique along with three re- 
ported novel image processing algorithms can be used as alternative medical imaging and diagnostic method.

Our infrared imaging method when compared to existing standard medical imaging carries many advantages, which are:

a) It is completely harmless to human body as compared to X-rays or MRI.Human body can be exposed to NIR for long durations as compared to ionizing radiation.

b) With enhanced IR imaging we produce much better visibility of tendons, soft tissues, blood vessels. It is well-known that tendons are not visible in X-rays and can be seen by MRI only.

c) Colorization of the images is expected to be of help to medical professionals.

\section{ACKNOWLEDGEMENTS}

The authors of the paper would like to express their gratitude for bleaching and colorizing of images performed by D. Sathe and D. Kulkarni.

\section{REFERENCES}

[1] Boas, D.A.; Brooks, D.H.; Miller, E.L.; DiMarzio, C.A.;Kilmer, M.; Gaudette, R.J.; Quan Zhang; Imaging the body with diffuse optical tomography, IEEE Signal Processing Magazine, 18, 6, pp: 57-75, 2001

[2] H. Dehghani, B. Pogue, J. Shudong, B. Brooksby, and K. Paulsen, Three-Dimensional Optical Tomography: Resolution in Small-Object Imaging, Appl. Opt.42, pp: 3117-3128, 2003

[3] Johnson, L.J.;Thyakor, N.;Hanley, D.; Study of near infrared imaging of a model of brain edema, Proc. of 18th IEEE Inter. Conf. in Medicine and Biology Society, 5, pp: 2107-2108, 2002
[4] Thiagarajah JR, Papadopoulos MC, Verkman AS, Noninvasive early detection of brain edema in mice by near-infrared light scattering, Jour. of Neurosci. Research, 2, 80, pp: 293-299, 2005

[5] Poellinger A, Burock S, Grosenick D, Hagen A, Lüdemann L, Diekmann F, Engelken F, Macdonald R, Rinneberg H, Schlag PM, Breast cancer: early- and late-fluorescence near-infrared imaging with indocyanine green--a preliminary study, Radiology, 2, 258, pp: 409-416, 2011

[6] Hebden J.C. and Rinneberg H., Optical mammography: Imaging and characterization of breast lesions by pulsed near-infrared laser light (OPTIMAMM), Phys. Medicine and Biology, 11, 50, 2005

[7] Dubinskaya V.,Eng Li, Rebrow L., Bykov V., Comparative study of the state of water in various human tissues, Bulletin of Experimental Biology and Medicine, Springer New York, 3, 144, pp: 294-297, 2009

[8] S.Mil'shtein, Infra-Red Scanning for Biomedical Applications, Scanning, 28, 5, pp274-277, 2005

[9] Frangioni J. V.,In vivo near-infrared fluorescence imaging, Current Opinion in Chemical Biology, 7, 5, pp: 626-634, 2003

[10] Rafael C. Gonzalez, Richard E. Woods, Steven L. Eddins, Digital Image Processing Using MATLAB, Prentice Hall, 2003

[11] Guan, L.; Ward, R.K.;Restoration of randomly blurred images by the Wiener filter, IEEE Trans. on Acoustics, Speech and Signal Processing, 4, 37, pp: 589-592, 2002

[12] Lingyu W., LeedhamG.,Near-and-Far-Infrared Imaging for Vein Pattern Biometrics, Proc. of IEEE Inter. Conf. on Video and Signal based Survelliance, 2006

[13] Wang L., Leedham G., A thermal hand vein pattern verification system,Pattern Recognition and Image Analysis, Lecture Notes in Computer Science, pp: 58-65, Springer, 2005

[14] Fantini S., Franceschini M.A., Handbook of Optical Biomedical Diagnostics, chapter 7. SPIE Press, Bellingham, WA, 2002 